\title{
Review Article \\ Recent Progress in Research on the Pathogenesis of Pulmonary Thromboembolism: An Old Story with New Perspectives
}

\author{
Chao Yan, Xiaohua Wang, Hua Su, and Kejing Ying \\ Department of Respiratory Medicine, Sir Run Run Shaw Hospital, School of Medicine, Zhejiang University, \\ Hangzhou 310016, China \\ Correspondence should be addressed to Kejing Ying; yingkejing@163.com
}

Received 17 November 2016; Revised 26 February 2017; Accepted 27 March 2017; Published 6 April 2017

Academic Editor: Salvatore Battaglia

Copyright (C) 2017 Chao Yan et al. This is an open access article distributed under the Creative Commons Attribution License, which permits unrestricted use, distribution, and reproduction in any medium, provided the original work is properly cited.

Pulmonary thromboembolism (PTE) is part of a larger clinicopathological entity, venous thromboembolism. It is also a complex, multifactorial disorder divided into four major disease processes including venous thrombosis, thrombus in transit, acute pulmonary embolism, and pulmonary circulation reconstruction. Even when treated, some patients develop chronic thromboembolic pulmonary hypertension. PTE is also a common fatal type of pulmonary vascular disease worldwide, but earlier studies primarily focused on the pathological changes in the blood component of the disease. With contemporary advances in molecular and cellular biology, people are becoming increasingly aware of coagulation pathways, the function of vascular smooth muscle cells, microparticles, and the inflammatory pathways that play key roles in PTE. Combined hypoxia and immune research has revealed that PTE should be regarded as a class of complex diseases caused by multiple factors involving the vascular microenvironment and vascular cell dysfunction.

\section{Introduction}

Venous thromboembolism (VTE), a combination of deep venous thrombosis (DVT) and pulmonary embolism (PE), is a major cause of morbidity and death in patients worldwide. $\mathrm{PE}$ is a common and potentially fatal disease that is caused by a perfusion defect due to an embolus blocking blood flow in the lungs [1]. VTE comprises all types of venous thrombosis in the various compartments, whether superficial or in the deep veins. PTE is also regarded as an acute complication of DVT [2]. The incidence of VTE is 1:1000 per year, and that of PE is approximately 50 in 100,000 per year in Europe $[3,4]$. Similarly, in the USA it affects an estimated number of 900,000 people each year, resulting in large numbers of hospitalizations and approximately 300,000 deaths [5].

Clinically, based on the classification of patients with acute PE based on early mortality risk, patients with highrisk PE (presenting with shock or hypotension) need to receive reperfusion therapy with thrombolysis, and low-risk (pulmonary embolism severity index [PESI] classes I-II or simplified pulmonary embolism severity index [sPESI] $=0$ ) and intermediate-risk (PESI classes III-IV or sPESI $\geq 1$ ) PEs are usually treated with anticoagulation and thrombolysis therapies to reconstruct the normal pulmonary hemodynamics [6]. The process of thrombolysis depends on two methods of clearing blood clots: thrombus dissolution by the endogenous fibrinolytic system and thrombus recanalization by the vascular microenvironment $[7,8]$. In addition to traditional anticoagulants (e.g., heparin, warfarin) [9], novel anticoagulant drugs (e.g., dabigatran, rivaroxaban) and new medical materials have also played an important role in the prevention and treatment of PTE [10, 11]. However, confusion still exists for certain patients. Even after formal treatment, such as continuous anticoagulant therapy for 3-6 months, normal pulmonary circulatory function cannot be reconstructed after PTE. These patients often end up with chronic thromboembolic pulmonary hypertension (CTEPH) [12].

In 1856, Virchow concluded that vessel wall injury, blood stasis, and hypercoagulability are the three synergic abnormalities that cause VTE [13]. The critical event in venous thrombosis is thrombin generation from the coagulation 
pathways, initialized by activated tissue factor (TF) [14]. In contrast, thrombus elimination is the result of a long-term interaction between the fibrinolysis system and the vascular microenvironment $[15,16]$. Many discoveries from recent studies have shown that the activation and signal transduction of protease activated receptors (PARs), pulmonary artery smooth muscle cells (PASMCs) responses to hypoxia, cellcell interactions mediated by extracellular vesicles (EVs), such as microparticles (MPs), and inflammation are the diverse mechanisms associated with the occurrence and development of PTE. This review discusses these potential mechanisms and aims to elucidate the detailed pathophysiology of PTE.

\section{PARs: Activation and Venous Thrombosis}

The initial event of PTE is venous thrombosis [14]. As a result, $\mathrm{PE}$ is considered an acute complication of DVT, and postthrombotic syndrome (PTS) is a long-term complication of DVT [17]. Therefore, a close relationship has been observed between PTE and coagulatory function. PARs serve as a significant driving force in the coagulation cascade and signal transduction pathways [18-20], and they may play an extremely important role in the process of PTE. PARs are seven-transmembrane G-protein-coupled receptors and include four family members, named PAR1-PAR4 [21]. PAR1, PAR3, and PAR4 are mainly cleaved and activated by thrombin, which acts as a key serine protease in the coagulation cascade [22-24]. However, PAR2 is a receptor for mast cell tryptase, coagulation factors VIIa and Xa, and trypsin $[25,26]$. This family is widely distributed throughout various organs of the body, such as the cardiovascular system, respiratory system, nervous system, and renal system [27]. Particularly, in the circulation system, it can promote the activation of platelets [28] or endothelial cells [29] and TF regulation in the pathological process [30]. PAR4-deficient mice are protected against thromboplastininduced PE [31]. However, it is important to note that there are species differences in the expression of PARs in platelets. For example, rat and mice platelets lack PAR1 $[32,33]$.

The activation of PARs requires proteolytic cleavage at the N-terminal extracellular domain, which generates a new $\mathrm{N}$-terminal domain that functions as a tethered ligand by binding intramolecularly to the receptor, triggering transmembrane signaling [18]. PAR-agonist peptides (PARAPs) are structurally similar to the tethered ligands and can activate PARs independently of protease activity and receptor cleavage [26]. Activated PARs initiate cell signaling via the recruitment of heterotrimeric $G$ proteins, including Gi, G12/13, and Gq [21]. Subsequently, downstream cellular responses lead to nociception, inflammation, cell migration, and proliferation, which often occur in pulmonary diseases [34].

PARs are expressed on both endothelial cells (ECs) and vascular smooth muscle cells (VSMCs). ECs mainly express PAR1, and other types of PARs are also present in very low amounts [35]. To represent typical vascular endothelial cells
(VECs), human umbilical vein endothelial cells (HUVECs) and pulmonary artery endothelial cells (PAECs) are used to study the relationship between ECs and coagulation function $[36,37]$. It has been demonstrated that TF-FVIIaXa complexes can transmit signals by PAR1 and TF-FVIIa complexes directly have a function that activates PAR2 [38]. These significant events occur during the process of clot formation and increased pulmonary vascular resistance in PTE.

However, studies suggest that the activation of TF and PARs is far more complex than once thought [39]. After specific peptide binding and activation, PAR1 and PAR2, which are expressed in HUVECs, and TF, which is expressed in ECs, can be induced by the redox-sensitive signaling pathway. The key signaling molecules of the common pathway are reactive oxygen species (ROS) generated by the mitochondrial electron transport chain and simultaneously activated ERK1/2 and MAPKp38. Mitochondrial complex III is mostly involved in the generation of ROS induced by PAR1 and PAR2. Researchers have also discovered that thrombin and PAR2-AP have a similar pharmacological activity to induce the production of TF mediated by mitochondrial complexes I and III [39, 40]. This finding contradicts the previous theory that the TF-VIIa complex activates PARs signal transduction independently [38]. Low concentrations of thrombin, which accumulates in the valve pocket, and local prolonged hypoxia caused by blood stasis are the two major characteristics of the initiation process of venous thrombosis $[41,42]$. In addition, the expression of TF at the embolism site is not significantly increased [43]. A reasonable assumption is that if TF is activated strongly and immediately on VECs through mROS, which are induced by thrombin or hypoxia in acute $\mathrm{PE}$, then it may play a central role in the triggering and spread of thrombi. This mechanism can explain the strengthened process of thrombosis in the acute phase of PTE. It also demonstrates that activation and regulation of TF and PARs are bidirectional.

Previous studies have shown that venous thrombi are mainly composed of fibrin and red blood cells [44, 45], and the role and function of platelets in VTE are rarely implicated. However, many recent studies show that platelets are a very important factor in thromboembolic disease. It was noted that venous thrombi contain platelets, and platelet activation is associated with thrombus initiation and propagation [46, 47]. PARs are important in multiple regulation pathways of platelet activation and directly or indirectly affect the progression of PTE. It is known that PAR1 can mediate the activation of human platelets by thrombin at low concentrations, but PAR4 plays a similar role at high thrombin concentrations if PAR1 is absent [18]. Recent research has shown that Prohibitin 1 (PHB1), expressed on the platelet membrane, has the function of microcontrol action on the PAR1 signaling pathway [48]. Experiments have confirmed that PHB1 is a type of membrane protein from human platelets that participates in the pathological activation of PAR1 and is induced by low concentrations of thrombin in PAR1-mediated platelet aggregation. Once PAR1 in HUVECs is activated, PHB1 also has an impact on the degradation and internalization of PAR1 [49]. 


\section{Functional Proteins in PASMCs: A Link between Pulmonary Vasoconstriction and Hypoxia}

The pulmonary vascular wall consists of three layers: the adventitia, media, and intima [50]. PASMCs located in the media are highly specialized cells. Differentiated SMCs (also called the "contractile phenotype") differ from other types of blood vessel cells, and their proliferation and synthesis abilities are inhibited [15, 51]. Under the effect of oxygen concentrations, PASMCs maintain vascular tension through the regulation of contraction and relaxation [52,53]. They can express proteins associated with contraction function [54] and ion channels that participate in the process of pulmonary vasoconstriction $[55,56]$.

Arterial hypoxemia, caused by the mismatching of ventilation and perfusion, is due to a thrombus obstructing the pulmonary artery or its branches in acute PE [57]. Hypoxic pulmonary vasoconstriction (HPV) can also elevate the pulmonary artery pressure (PAP) by increasing the pulmonary vascular resistance [58]. The exact mechanism has not been fully clarified, but PASMCs play an important role in HPV. Previous studies have suggested that oxygen concentrations can be "sensed" by mitochondria [40] or NADPH oxidases [59] from PASMCs related to the alteration of ROS. There is a debate in the literature regarding whether ROS levels increase or decrease during hypoxia [60-62]. However, it is known that ROS has the function of regulating the $\mathrm{K}^{+}$ and $\mathrm{Ca}^{2+}$ channels in PASMCs $[63,64]$. Redox-sensitive $\mathrm{K}^{+}$channels were found to be inhibited under hypoxia, and membrane potential changes and voltage-gated L-type $\mathrm{Ca}^{2+}$ channels were activated. Elevated intracellular $\mathrm{Ca}^{2+}$ concentrations from the internal release of calcium, or the influx of extracellular calcium, produce signals that trigger PASMCs contraction by activating actin and myosin.

Presently, the theory regarding the precise mechanism of HPV remains unclear. For example, the functions of actin-associated proteins in PTE are largely unknown. We have shown that hypoxia can regulate the production of two actin-associated proteins through the hypoxia inducible factor (HIF) pathway in human or rat PASMCs. HIF- $1 \alpha$ can induce the expression of the capping protein CapG, and HIF- $2 \alpha$ can induce the expression of transgelin (SM22) $[65,66]$. CapG is an actin regulatory protein that can modulate actin length by binding and capping the end of actin filaments in a $\mathrm{Ca}^{2+}$ - and polyphosphoinositide-dependent manner [67]. Transgelin can participate in the organization of actin distribution by interacting with actin and plays an important role in the regulation of PASMCs contraction in a $\mathrm{Ca}^{2+}$-independent manner $[68,69]$. The upregulation of these two proteins contributes to the increased motility and contraction of PASMCs under acute or sustained exposure to low-oxygen environments. These observations indicate that the two oxygen-sensing pathways that are dependent on ROS or HIF may both play a significant role in pulmonary vasoconstriction after $\mathrm{PE}$.

Acute hypoxia in PTE causes clinical manifestations, including vascular contraction, alveolar hypoxia, and the increased blood coagulation activity [42, 70]. However, unlike acute hypoxia, sustained hypoxia is more common in a variety of pulmonary vascular diseases [71]. The upregulation of transgelin and CapG enhances the migratory ability of PASMCs during the process of vascular remodeling caused by chronic hypoxia $[66,72]$. In future studies, it would be interesting to focus on the differences in the mechanism between CTEPH associated with PTE and hypoxic pulmonary hypertension $(\mathrm{HPH})$, due to lung disease and/or hypoxia. Hypoxia also overcomes the balance of ACE/ACE2 expression levels by HIF-1 $\alpha$ [73] and plays key roles in promoting the contraction and proliferation of PASMCs by inhibiting the ACE2Ang(1-7)-Mas axis. The administration of recombinant ACE2 suppresses the pulmonary vasoconstriction response to acute hypoxia that occurs in pulmonary hypertension $(\mathrm{PH})$ related to high altitude [74], which is similar to the symptoms of acute PE. Moreover, the functions of the contraction, secretion, growth, and migration of PASMCs greatly affect thrombus dissolution and recanalization, which are closely associated with the prognosis of an individual patient after acute PE.

\section{Microparticles: A Potential Biomarker for PTE}

EVs are nanosized, membrane-limited vesicles released from cells that participate in cell-cell communication [75]. Several types of cells are capable of releasing EVs, which can transport DNA, RNA, lipids, and proteins [76] by shedding vesicles from their plasma membrane. Due to the release of EVs from cells throughout the body, they can also be detected in diverse body fluids [75] and in cell culture supernatants [77]. Cells produce different subtypes of EVs that vary in size, including microvesicles (MVs)/microparticles (MPs), exosomes, oncosomes, and apoptotic bodies [78]. MVs and exosomes are generated by normal and cancer cells [76]. The mechanism of intercellular information transmission by these two major types of EVs has also been extensively studied. MVs that contain cytoplasmic cargos are $0.1-1 \mu \mathrm{m}$ in diameter and come directly from the plasma membrane $[78,79]$. MPs and exosomes can be released from VECs and VSMCs induced by hypoxia [80], shear stress [81], or cytokines [82] during the disease process. Therefore, they are regarded as potential biomarkers for the diagnosis or prognosis of PTE and provide a new treatment strategy in addition to anticoagulation and thrombolysis [83].

MPs seem to be associated with a hypercoagulable state, which predisposes a person to thrombosis but does not determine its occurrence. The levels of circulating tissue factor bearing MP $\left(\mathrm{TF}^{+} \mathrm{MPs}\right)$ [84] or phosphatidylserine $\left(\mathrm{PS}^{+}\right)$and lactadherin ${ }^{+} \mathrm{MPs}$ [85] are higher in patients with a hypercoagulable status than in control subjects. Elevated circulating MP-TF activity is associated with thrombosis and worsened survival in patients with pancreaticobiliary cancers (PBCs) [86]. Increased levels of glial-derived and/or $\mathrm{TF}^{+} \mathrm{MPs}$ are also noted in glioblastoma multiforme (GBM) patients both before and even more so after the neoplasm is treated, suggesting a contribution of $\mathrm{TF}^{+} / \mathrm{GFAP}-\mathrm{MPs}$ to the 
risk of VTE [87]. Watts and colleagues [88] demonstrated that MP protein composition changes and the proteins involved in clot formation increase during PE compared with controls. The process of acute PE in a rat model induced by injection with polystyrene microspheres also showed that the proteins inside MPs have prothrombotic characteristics related to fibronectin, fibrinogen, and von Willebrand factor. However, in another study, Garcia Rodriguez and colleagues [89] demonstrated that plasma MP-TF activity in patients presenting with clinically suspected PE was not associated with confirmed PE. In both groups of patients presenting with symptoms of PE, the median MP-TF activities were significantly higher than those in the healthy controls, especially in patients with the presence of active cancer or cardiovascular disease. These findings suggest that the high MP-TF activity levels found in cancer patients with acute VTE originate from aggressive tumour cells rather than an acute thromboembolic event.

Valuable biomarker candidates must possess specificity, sensitivity, timeliness, and a biological gradient. Associations between elevated MPs levels and an increased risk of VTE have been found in patients with underlying diseases such as high-grade glioma [90] or in patients undergoing in vitro fertilization [91] to treat infertility. However, confusion and contradictions are still present in clinical studies [92, 93]. Further research should focus on the following problems:

(I) The limitations of current assays in measuring MPs may be divided into sizing, probing, and counting [94]. The resultant difference between flow cytometry and other methods such as enzyme-linked immunosorbent assays is a widespread problem because a standardized conversion between the two methods is difficult and lacking.

(II) Some studies have shown that PS- or TF-dependent procoagulant activity is consistent with a hypercoagulable status and the occurrence of VTE [85, 95]. Further studies are necessary to identify the diagnostic value and clinical significance of different procoagulant markers from MPs.

(III) MPs are derived from various cells, including blood cells, ECs, SMCs, and malignant cells [96]. Mesenchymal stem cells, which are applied to biotherapy, can also release MVs [97], and PTE is one of the most serious complications during the treatment procedures [98]. Thus, distinguishing the source of MPs is one of the core problems associated with using MPs as a biomarker to diagnose or assess the prognosis of PTE.

(IV) In the process of collecting and detecting blood samples, researchers often overlook the fact that different treatment methods or drug applications involving anticoagulants have potential effects on the production or function of $\mathrm{TF}^{+} \mathrm{MPs}$ [99]. Antitumor therapies, such as chemotherapy, may also increase the level and/or activity of $\mathrm{TF}^{+} \mathrm{MP}[100,101]$.

\section{Inflammation: A Close Association with the Occurrence of PTE}

Under the condition of multiple stimuli such as hypoxia or trauma, inflammation is a trigger for pulmonary endothelial dysfunction and platelet activation [102, 103]. Evidence has implicated the presence of inflammation with acute PE, including the observation that the white blood cell (WBC) count and neutrophil-to-lymphocyte ratio (NLR) are associated with short-term outcomes in PE patients. Venetz et al. [104] demonstrated that patients with an elevated WBC count $\left(>9.8 \times 10^{9} / \mathrm{L}\right)$ have a significantly higher 30 -day mortality than patients who do not, after adjustment for thrombolytic therapy and for patient- and hospital-related confounders. Previous studies [105] have shown that an elevated WBC count may be a marker for hypercoagulability and that the WBC count correlates with levels of fibrinogen, factor VII, and factor VIII. A retrospective cohort study [106], including $667 \mathrm{PE}$ patients, further showed that leukocytosis and the systemic inflammatory response are prognostic factors for 30-day mortality after PE. Other researchers have also observed that the platelet-to-lymphocyte ratio (PLR) is an independent predictor of mortality in acute PE patients, which is significantly correlated with PESI scores [107]. Moreover, the NLR is considered a better independent predictor of in-hospital mortality and may be used for clinical risk classification because of its reliability in the distinction between massive and submassive embolism [108].

Furthermore, the key role of neutrophils in PTE has been confirmed by a series of experimental studies. Cytokineinduced neutrophil chemoattractant-1 (CINC-1) expression increased 18- and 24-fold at 6 and $18 \mathrm{~h}$ after PE, respectively, and an influx of neutrophils was observed, with a significant upregulation $18 \mathrm{~h}$ after PE [109]. Neutrophilic inflammation is also observed in the lungs in an acute PE model in which bronchoalveolar lavage-associated neutrophils showed an almost 6-fold increase in rats with severe PTE compared with controls or rats with moderate PTE [110]. These results show that neutrophils contribute to right ventricular dysfunction and lung damage in rat PE. However, there was a remarkable difference between acute PH caused by PTE and chronic PH caused by HPH or CTEPH [111]. Some scholars believe that inflammation is an important pathological process of right ventricular damage after PE and can indirectly lead to a poor clinical outcome [112].

An increasing number of studies have also found that patients with chronic inflammatory disorders of the airway, such as asthma and chronic obstructive pulmonary disease (COPD), are at high risk of PTE. In a population-based, case-control retrospective study [113] that included 909,638 individuals (429,962 males and 479,676 females) aged over 14 years and 55,500 (6.1\%) individuals suffering from asthma, the prevalence of $\mathrm{PE}$ was found to be substantially higher among asthmatic patients than in the nonasthmatic general population $(0.26 \%$ versus $0.17 \%)$. A retrospective study [114] that included 648 patients with asthma (283 with severe and 365 patients with mild-to-moderate asthma) showed an almost 9-fold higher risk of $\mathrm{PE}$ in patients with severe 


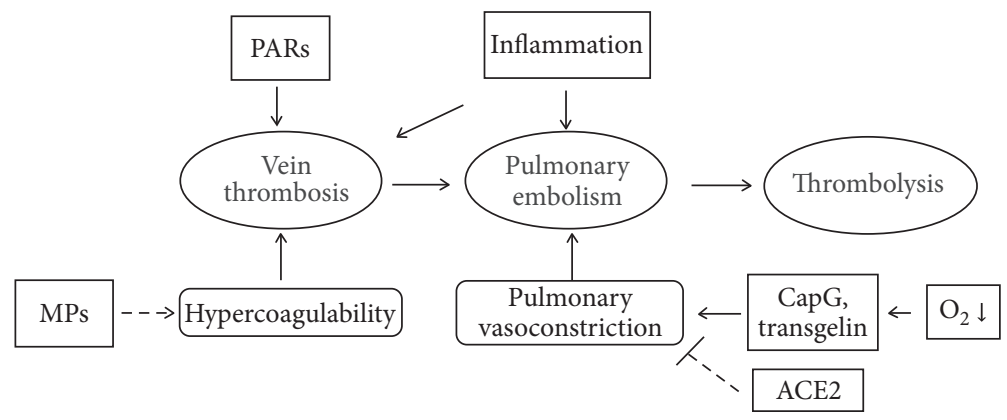

FIGURE 1: Protease activated receptors (PARs), pulmonary artery smooth muscle cells (PASMCs), microparticles (MPs), and inflammation are associated with pulmonary thromboembolism (PTE). Biomedical research has shown that PARs modulate thrombosis by activating ECs, and functional proteins (e.g., CapG, transgelin, and ACE2) in PASMCs have an important function in pulmonary vasoconstriction. Clinical research links MPs and hypercoagulability to venous thromboembolism. Epidemiological studies have shown that inflammation is a risk factor for PTE.

asthma and a 3.5-fold higher risk of PE in patients with mildto-moderate asthma than in the general population. Oral corticosteroid use was also found to be an independent risk factor for PE. In a large cohort study [115] that included 31,356 asthmatic patients and 125,157 nonasthmatic controls, the overall incidence rate of PE showed a 3.30-fold increase in the asthmatic patients compared with the nonasthmatic cohort (10.2 versus 3.09 per 100,000 person-years). One hypothesis is that asthma may reduce the process of clot retraction, which makes the thrombus stronger and more resistant to shear stresses and fibrinolysis. Recent studies have confirmed that asthma is associated with a significant inhibition of clot retraction [116]. The potential mechanism is that reactive nitrogen species produced in the lungs of asthmatic patients may reduce platelet contractility through the diminution of platelet energy production.

Several studies have also shown an increased prevalence of PE in COPD patients. COPD is associated with an increased risk of VTE, and PE presentation is more significantly associated with COPD patients than non-COPD patients (OR 1.64, 95\% CI 1.49-1.80) [117]. Specifically, COPD is associated with an increased risk of mortality $(10.8 \%$ versus $7.6 \%)$, minor bleeding (4.5\% versus $2.3 \%)$, and first VTE recurrences as $\mathrm{PE}(1.5 \%$ versus $1.1 \%)$ during the 3 -month follow-up. Similar to asthma, COPD patients present more frequently with PE than with DVT $[114,117]$. A retrospective population-based cohort study [118] using data retrieved from Taiwan's National Health Insurance Research Database (2000 to 2008), including 355,878 COPD patients and 355,878 comparison patients, shows that the prevalence of $\mathrm{PE}$ in COPD patients is 3.45 -fold higher than that in non-COPD patients and increases with age. Severely exacerbated COPD patients, especially those with immobility/obesity [119] or those requiring ICU admission [120], have an increased risk of PE.

The complex relationship between PTE and respiratory allergy/inflammatory diseases is partly attributed to various types of inflammatory cytokines and immune cells. Proinflammatory activity is one of the predominant features in interleukin family members [121], but some members have an anti-inflammatory function [122]. For example, M1- or M2- type macrophages play different roles in tissue damage and inflammatory responses [123]. More importantly, neutrophils are simultaneously involved in thrombosis and thrombolysis, which are two key processes in PTE. Neutrophil extracellular traps (NETs) are considered to be one of the basic structures of thrombosis [124], and the dissolution of NETs can also affect thrombus dissolution and recanalization [125]. Antiinflammatory treatment for PTE is not as simple as anticoagulation therapy, but it will be a focus of future studies because of its potential implications in PTE.

\section{Summary and Perspective}

PTE is more closely associated with coagulation function than lung cancer, pneumonia, and other common respiratory diseases. Virchow's Triad preliminarily revealed the internal relationships between coagulant function abnormality and pathological changes in the vessel wall, based on venous thrombosis, which is the initial event of pulmonary thromboembolism. Focusing on a molecular and cellular view, contemporary research reveals that the function of coagulation involving vascular endothelium in PTE is affected by hypoxia or inflammation, and developing an efficient test for the diagnosis and prognosis of PTE depends on the knowledge of the balance between prothrombotic and antithrombotic factors in the lung.

The aim of contemporary research is to understand the pathogenesis of PTE (Figure 1). Various laboratory studies have demonstrated the importance of PAR activation, hypoxia signaling pathways, and the generation of MPs. However, the inflammatory response is a link involving the above three events in PTE and may have an overall influence on signal transduction in the progression of the disease, depending on ECs or platelets $[8,126,127]$. Compared with traditional anticoagulation and thrombolysis therapy based on the theory of thrombosis, the activation and regulation of inflammatory pathways are complex. For example, inflammasomes are activated in diseases associated with sterile inflammation [128] and can sometimes be initiated by peptide secretions of the host at earlier stages of infection or trauma, to protect the host against microbial infection 
[129]. Some inflammatory factors also have a function as a two-way switch, and specific types of leukocytes, such as neutrophils, participate in the two important processes of thrombosis and thrombolysis in PTE. This finding suggests that immunization therapy for PTE is destined to be useful and that a purely anti-inflammatory treatment cannot be used, periodically, to suppress the inflammatory process. Inflammation and metabolism also have a very complex link. Extracellular ATP has a proinflammatory role in the process of infection, and this effect is produced by the activation of inflammasomes [130]. Additionally, hypoxia induces the caspase-1-mediated activation of the NALP3 inflammasome in chronic HPH [131].

Numerous clinical studies involving PTE have summarized the spectrum of risk factors associated with the occurrence and development of disease [132], and the prevention and treatment of PTE have a high rate of success based on current risk stratification. Similarly, the complex cellular and molecular mechanisms must also be explained through basic research which may generate new therapeutic strategies. Due to the improvements in animal models [133], research developments in the field of $\mathrm{PH}$ are more comprehensive and have a more specific theoretical depth than do those in the field of PTE. It is worth learning from the experiences gained from studies in $\mathrm{PH}$. In addition to anticoagulant and thrombolytic therapy, new targeted drugs and treatments must be perfected based on the progress of PTE animal models and research methods, as well as on integrating, the involvement of anticoagulants, procoagulants, fibrinolytics, and the immune system, rather than focusing solely on the thrombus.

\section{Conflicts of Interest}

The authors have no conflicts of interest to declare.

\section{Acknowledgments}

This work was supported by the National Natural Science Foundation of China [Grant no. 81270107] and the Medical Research Program of Zhejiang Province [Grant no. 2016KYB149].

\section{References}

[1] N. M. Di, E. N. Van, and H. R. Büller, "Deep vein thrombosis and pulmonary embolism," The Lancet, vol. 388, no. 10063, pp. 3060-3073, 2016.

[2] K. K. Søgaard, M. Schmidt, L. Pedersen, E. Horváth-Puhó, and H. T. Sørensen, "30-year mortality after venous thromboembolism a population-based cohort study," Circulation, vol. 130, no. 10, pp. 829-836, 2014.

[3] J. A. Heit, "Epidemiology of venous thromboembolism," Nature Reviews Cardiology, vol. 12, no. 8, pp. 464-474, 2015.

[4] A. T. Cohen, G. Agnelli, F. A. Anderson et al., "Venous thromboembolism (VTE) in Europe-the number of VTE events and associated morbidity and mortality," Thrombosis and Haemostasis, vol. 98, no. 4, pp. 756-764, 2007.
[5] G. E. Raskob, R. Silverstein, D. W. Bratzler, J. A. Heit, and R. H. White, "Surveillance for deep vein thrombosis and pulmonary embolism: recommendations from a national workshop," American Journal of Preventive Medicine, vol. 38, no. 4, supplement, pp. S502-S509, 2010.

[6] S. V. Konstantinides, A. Torbicki, G. Agnelli et al., "2014 ESC guidelines on the diagnosis and management of acute pulmonary embolism," European Heart Journal, vol. 35, no. 43, pp. 3033-3069, 2014.

[7] G. Piazza and S. Z. Goldhaber, "Fibrinolysis for acute pulmonary embolism," Vascular Medicine, vol. 15, no. 5, pp. 419$428,2010$.

[8] C. E. Evans, J. Humphries, K. Mattock et al., "Hypoxia and upregulation of hypoxia-inducible factor $1 \alpha$ stimulate venous thrombus recanalization," Arteriosclerosis, Thrombosis, and Vascular Biology, vol. 30, no. 12, pp. 2443-2451, 2010.

[9] V. F. Tapson, "Thrombolytic therapy for acute pulmonary embolism," Seminars in Thrombosis and Hemostasis, vol. 39, no. 4, pp. 452-458, 2013.

[10] P. P. Dobesh and J. Fanikos, "New oral anticoagulants for the treatment of venous thromboembolism: understanding differences and similarities," Drugs, vol. 74, no. 17, pp. 20152032, 2014.

[11] R. S. Smith, Z. Zhang, M. Bouchard et al., "Vascular catheters with a nonleaching poly-sulfobetaine surface modification reduce thrombus formation and microbial attachment," Science Translational Medicine, vol. 4, no. 153, pp. 2569-2575, 2012.

[12] I. M. Lang, R. Pesavento, D. Bonderman, and J. X.-J. Yuan, "Risk factors and basic mechanisms of chronic thromboembolic pulmonary hypertension: a current understanding," European Respiratory Journal, vol. 41, no. 2, pp. 462-468, 2013.

[13] P. H. Reitsma, H. H. Versteeg, and S. Middeldorp, "Mechanistic view of risk factors for venous thromboembolism," Arteriosclerosis, Thrombosis, and Vascular Biology, vol. 32, no. 3, pp. 563568, 2012.

[14] A. P. Owens III and N. Mackman, "Tissue factor and thrombosis: the clot starts here," Thrombosis and Haemostasis, vol. 104, no. 3, pp. 432-439, 2010.

[15] S. Alias and I. M. Lang, "Coagulation and the vessel wall in pulmonary embolism," Pulmonary Circulation, vol. 3, no. 4, pp. 728-738, 2013.

[16] M. K. Jezovnik and P. Poredos, "Factors influencing the recanalisation rate of deep venous thrombosis," International Angiology, vol. 31, no. 2, pp. 169-175, 2012.

[17] F. A. Klok, T. van der Hulle, P. L. den Exter, M. Lankeit, M. V. Huisman, and S. Konstantinides, "The post-PE syndrome: a new concept for chronic complications of pulmonary embolism," Blood Reviews, vol. 28, no. 6, pp. 221-226, 2014.

[18] S. R. Coughlin, “Thrombin signalling and protease-activated receptors," Nature, vol. 407, no. 6801, pp. 258-264, 2000.

[19] A. R. Rezaie, "Protease-activated receptor signalling by coagulation proteases in endothelial cells," Thrombosis and Haemostasis, vol. 112, no. 5, pp. 876-882, 2014.

[20] T. A. McEachron, R. Pawlinski, K. L. Richards, F. C. Church, and N. Mackman, "Protease-activated receptors mediate crosstalk between coagulation and fibrinolysis," Blood, vol. 116, no. 23, pp. 5037-5044, 2010.

[21] U. J. Soh, M. R. Dores, B. Chen, and J. Trejo, "Signal transduction by protease-activated receptors," British Journal of Pharmacology, vol. 160, no. 2, pp. 191-203, 2010. 
[22] T.-K. H. Vu, D. T. Hung, V. I. Wheaton, and S. R. Coughlin, "Molecular cloning of a functional thrombin receptor reveals a novel proteolytic mechanism of receptor activation," Cell, vol. 64, no. 6, pp. 1057-1068, 1991.

[23] H. Ishihara, A. J. Connolly, D. Zeng et al., "Protease-activated receptor 3 is a second thrombin receptor in humans," Nature, vol. 386, no. 6624, pp. 502-506, 1997.

[24] W.-F. Xu, H. Andersen, T. E. Whitmore et al., "Cloning and characterization of human protease-activated receptor 4," Proceedings of the National Academy of Sciences of the United States of America, vol. 95, no. 12, pp. 6642-6646, 1998.

[25] S. Nystedt, K. Emilsson, C. Wahlestedt, and J. Sundelin, "Molecular cloning of a potential proteinase activated receptor," Proceedings of the National Academy of Sciences of the United States of America, vol. 91, no. 20, pp. 9208-9212, 1994.

[26] M. N. Adams, R. Ramachandran, M.-K. Yau et al., "Structure, function and pathophysiology of protease activated receptors," Pharmacology and Therapeutics, vol. 130, no. 3, pp. 248-282, 2011.

[27] Y. Bulani and S. S. Sharma, "Therapeutic potential of targeting protease activated receptors in cardiovascular diseases," Current Pharmaceutical Design, vol. 21, no. 30, pp. 4392-4399, 2015.

[28] Z. Li, M. K. Delaney, K. A. O’Brien, and X. Du, "Signaling during platelet adhesion and activation," Arteriosclerosis, Thrombosis, and Vascular Biology, vol. 30, no. 12, pp. 2341-2349, 2010.

[29] M. Riewald, R. J. Petrovan, A. Donner, B. M. Mueller, and W. Ruf, "Activation of endothelial cell protease activated receptor 1 by the protein C pathway," Science, vol. 296, no. 5574, pp. 1880$1882,2002$.

[30] E. Camerer, W. Huang, and S. R. Coughlin, "Tissue factor- and factor X-dependent activation of protease-activated receptor 2 by factor VIIa," Proceedings of the National Academy of Sciences of the United States of America, vol. 97, no. 10, pp. 5255-5260, 2000.

[31] E. J. Weiss, J. R. Hamilton, K. E. Lease, and S. R. Coughlin, "Protection against thrombosis in mice lacking PAR3," Blood, vol. 100, no. 9, pp. 3240-3244, 2002.

[32] M. Kogushi, T. Matsuoka, H. Kuramochi et al., "Oral administration of the thrombin receptor antagonist E5555 (atopaxar) attenuates intimal thickening following balloon injury in rats," European Journal of Pharmacology, vol. 666, no. 1-3, pp. 158164, 2011.

[33] F. Nadal-Wollbold, A. Bocquet, T. Bourbon, R. Létienne, and B. Le Grand, "Protease-activated receptor 1 antagonists prevent platelet aggregation and adhesion without affecting thrombin time," European Journal of Pharmacology, vol. 644, no. 1-3, pp. 188-194, 2010.

[34] R. J. José, A. E. Williams, and R. C. Chambers, "Proteinaseactivated receptors in fibroproliferative lung disease," Thorax, vol. 69, no. 2, pp. 190-192, 2014.

[35] R. T. Abdallah, J. S. Keum, H. M. El-Shewy et al., "Plasma kallikrein promotes epidermal growth factor receptor transactivation and signaling in vascular smooth muscle through direct activation of protease-activated receptors," The Journal of Biological Chemistry, vol. 285, no. 45, pp. 35206-35215, 2010.

[36] S. Gödecke, C. Roderigo, C. R. Rose, B. H. Rauch, A. Gödecke, and J. Schrader, "Thrombin-induced ATP release from human umbilical vein endothelial cells," American Journal of Physiology-Cell Physiology, vol. 302, no. 6, pp. C915-C923, 2012.

[37] J.-S. Bae, Y.-U. Kim, M.-K. Park, and A. R. Rezaie, "Concentration dependent dual effect of thrombin in endothelial cells via
Par-1 and Pi3 kinase," Journal of Cellular Physiology, vol. 219, no. 3, pp. 744-751, 2009.

[38] N. Mackman, "The many faces of tissue factor," Journal of Thrombosis and Haemostasis, vol. 7, supplement 1, pp. 136-139, 2009.

[39] C. Banfi, M. Brioschi, S. S. Barbieri et al., "Mitochondrial reactive oxygen species: a common pathway for PAR1- and PAR2-mediated tissue factor induction in human endothelial cells," Journal of Thrombosis and Haemostasis, vol. 7, no. 1, pp. 206-216, 2009.

[40] R. B. Hamanaka and N. S. Chandel, "Mitochondrial reactive oxygen species regulate hypoxic signaling," Current Opinion in Cell Biology, vol. 21, no. 6, pp. 894-899, 2009.

[41] D. A. Manly, J. Boles, and N. MacKman, "Role of tissue factor in venous thrombosis," Annual Review of Physiology, vol. 73, pp. 515-525, 2011.

[42] E. G. Bovill and A. Van Der Vliet, "Venous valvular stasisassociated hypoxia and thrombosis: what is the link?" Annual Review of Physiology, vol. 73, pp. 527-545, 2011.

[43] J.-X. Zhang, Y.-L. Chen, Y.-L. Zhou, Q.-Y. Guo, and X.-P. Wang, "Expression of tissue factor in rabbit pulmonary artery in an acute pulmonary embolism model," World Journal of Emergency Medicine, vol. 5, no. 2, pp. 144-147, 2014.

[44] N. Mackman, "Triggers, targets and treatments for thrombosis," Nature, vol. 451, no. 7181, pp. 914-918, 2008.

[45] M. M. Aleman, B. L. Walton, J. R. Byrnes, and A. S. Wolberg, "Fibrinogen and red blood cells in venous thrombosis," Thrombosis Research, vol. 133, supplement 1, pp. S38-S40, 2014.

[46] N. Mackman, "New insights into the mechanisms of venous thrombosis," The Journal of Clinical Investigation, vol. 122, no. 7, pp. 2331-2336, 2012.

[47] S. Montoro-García, M. Schindewolf, S. Stanford, O. H. Larsen, and T. Thiele, "The role of platelets in venous thromboembolism," Seminars in Thrombosis and Hemostasis, vol. 42, no. 3, pp. 242-251, 2016.

[48] Y. Zhang, Y. Wang, Y. Xiang, W. Lee, and Y. Zhang, "Prohibitins are involved in protease-activated receptor 1-mediated platelet aggregation," Journal of Thrombosis and Haemostasis, vol. 10, no. 3, pp. 411-418, 2012.

[49] Y. J. Wang, X. L. Guo, S. A. Li et al., "Prohibitin is involved in the activated internalization and degradation of proteaseactivated receptor 1," Biochimica et Biophysica Acta-Molecular Cell Research, vol. 1843, no. 7, pp. 1393-1401, 2014.

[50] O. Pak, A. Aldashev, D. Welsh, and A. Peacock, "The effects of hypoxia on the cells of the pulmonary vasculature," European Respiratory Journal, vol. 30, no. 2, pp. 364-372, 2007.

[51] D. Aoshima, T. Murata, M. Hori, and H. Ozaki, "Timedependent phenotypic and contractile changes of pulmonary artery in chronic hypoxia-induced pulmonary hypertension," Journal of Pharmacological Sciences, vol. 110, no. 2, pp. 182-190, 2009.

[52] D. K. Singh, J. Sarkar, A. Raghavan, S. P. Reddy, and J. U. Raj, "Hypoxia modulates the expression of leucine zipper-positive MYPT1 and its interaction with protein kinase G and Rho kinases in pulmonary arterial smooth muscle cells," Pulmonary Circulation, vol. 1, no. 4, pp. 487-498, 2011.

[53] K. R. Olson, N. L. Whitfield, S. E. Bearden et al., "Hypoxic pulmonary vasodilation: a paradigm shift with a hydrogen sulfide mechanism," American Journal of Physiology-Regulatory Integrative and Comparative Physiology, vol. 298, no. 1, pp. R51R60, 2010. 
[54] R. Yamin and K. G. Morgan, "Deciphering actin cytoskeletal function in the contractile vascular smooth muscle cell," Journal of Physiology, vol. 590, no. 17, pp. 4145-4154, 2012.

[55] W. S. Park, A. L. Firth, J. Han, and E. A. Ko, "Patho, physiological roles of voltage-dependent $\mathrm{K}+$ channels in pulmonary arterial smooth muscle cells," Journal of Smooth Muscle Research, vol. 46, no. 2, pp. 89-105, 2010.

[56] A. L. Firth, C. V. Remillard, O. Platoshyn, I. Fantozzi, E. A. Ko, and J. X. Yuan, "Functional ion channels in human pulmonary artery smooth muscle cells: voltage-dependent cation channels," Pulmonary Circulation, vol. 1, no. 1, pp. 48-71, 2011.

[57] G. Piazza and S. Z. Goldhaber, "Management of submassive pulmonary embolism," Circulation, vol. 122, no. 11, pp. 1124$1129,2010$.

[58] N. Sommer, I. Strielkov, O. Pak, and N. Weissmann, "Oxygen sensing and signal transduction in hypoxic pulmonary vasoconstriction," European Respiratory Journal, vol. 47, no. 1, pp. 288-303, 2016.

[59] A. Konior, A. Schramm, M. Czesnikiewicz-Guzik, and T. J. Guzik, "NADPH oxidases in vascular pathology," Antioxidants and Redox Signaling, vol. 20, no. 17, pp. 2794-2814, 2014.

[60] B. Fuchs, N. Sommer, A. Dietrich et al., "Redox signaling and reactive oxygen species in hypoxic pulmonary vasoconstriction," Respiratory Physiology and Neurobiology, vol. 174, no. 3, pp. 282-291, 2010.

[61] G. B. Waypa, J. D. Marks, R. Guzy et al., "Hypoxia triggers subcellular compartmental redox signaling in vascular smooth muscle cells," Circulation Research, vol. 106, no. 3, pp. 526-535, 2010.

[62] A. Olschewski and E. K. Weir, "Redox regulation of ion channels in the pulmonary circulation," Antioxidants and Redox Signaling, vol. 22, no. 6, pp. 465-485, 2015.

[63] R. Moudgil, E. D. Michelakis, and S. L. Archer, "Hypoxic pulmonary vasoconstriction," Journal of Applied Physiology, vol. 98, no. 1, pp. 390-403, 2005.

[64] F. Veit, O. Pak, R. P. Brandes, and N. Weissmann, "Hypoxiadependent reactive oxygen species signaling in the pulmonary circulation: focus on ion channels," Antioxidants and Redox Signaling, vol. 22, no. 6, pp. 537-552, 2015.

[65] R. Zhang, L. Zhou, Q. Li, J. Liu, W. Yao, and H. Wan, "Upregulation of two actin-associated proteins prompts pulmonary artery smooth muscle cell migration under hypoxia," American Journal of Respiratory Cell and Molecular Biology, vol. 41, no. 4, pp. 467-475, 2009.

[66] R. Zhang, L. Shi, L. Zhou et al., "Transgelin as a therapeutic target to prevent hypoxic pulmonary hypertension," American Journal of Physiology_Lung Cellular and Molecular Physiology, vol. 306, no. 6, pp. L574-L583, 2014.

[67] C. Pellieux, A. Desgeorges, C. H. Pigeon et al., “Cap G, a gelsolin family protein modulating protective effects of unidirectional shear stress," The Journal of Biological Chemistry, vol. 278, no. 31, pp. 29136-29144, 2003.

[68] H. D. Je and U. D. Sohn, "SM22 $\alpha$ is required for agonist-induced regulation of contractility: evidence from SM22 $\alpha$ knockout mice," Molecules and Cells, vol. 23, no. 2, pp. 175-181, 2007.

[69] C. B. Marshall, R. D. Krofft, M. J. Blonski et al., "Role of smooth muscle protein SM22 $\alpha$ in glomerular epithelial cell injury," American Journal of Physiology-Renal Physiology, vol. 300, no. 4, pp. 1026-1042, 2011.

[70] M. Toba, T. Nagaoka, Y. Morio et al., "Involvement of Rho kinase in the pathogenesis of acute pulmonary embolisminduced polystyrene microspheres in rats," American Journal of
Physiology_Lung Cellular and Molecular Physiology, vol. 298, no. 3, pp. L297-L303, 2010.

[71] N. F. Voelkel, S. Mizuno, and H. J. Bogaard, "The role of hypoxia in pulmonary vascular diseases: a perspective," American Journal of Physiology -Lung Cellular and Molecular Physiology, vol. 304, no. 7, pp. L457-L465, 2013.

[72] X. Xu, H. Hu, X. Wang et al., "Involvement of CapG in proliferation and apoptosis of pulmonary arterial smooth muscle cells and in hypoxia-induced pulmonary hypertension rat model," Experimental Lung Research, vol. 42, no. 3, pp. 142-153, 2016.

[73] R. Zhang, Y. Wu, M. Zhao et al., "Role of HIF-1 $\alpha$ in the regulation ACE and ACE2 expression in hypoxic human pulmonary artery smooth muscle cells," American Journal of Physiology_Lung Cellular and Molecular Physiology, vol. 297, no. 4, pp. L631-L640, 2009.

[74] A. Kleinsasser, I. Pircher, B. Treml et al., "Recombinant angiotensin-converting enzyme 2 suppresses pulmonary vasoconstriction in acute hypoxia," Wilderness and Environmental Medicine, vol. 23, no. 1, pp. 24-30, 2012.

[75] G. Raposo and W. Stoorvogel, "Extracellular vesicles: exosomes, microvesicles, and friends," Journal of Cell Biology, vol. 200, no. 4, pp. 373-383, 2013.

[76] V. R. Minciacchi, M. R. Freeman, and D. Di Vizio, "Extracellular vesicles in cancer: exosomes, microvesicles and the emerging role of large oncosomes," Seminars in Cell and Developmental Biology, vol. 40, pp. 41-51, 2015.

[77] M. Franquesa, M. J. Hoogduijn, E. Ripoll et al., "Update on controls for isolation and quantification methodology of extracellular vesicles derived from adipose tissue mesenchymal stem cells," Frontiers in Immunology, vol. 5, article 525, 2014.

[78] B. Gyorgy, T. G. Szabo, M. Pasztoi et al., "Membrane vesicles, current state-of-the-art: emerging role of extracellular vesicles," Cellular and Molecular Life Sciences, vol. 68, no. 16, pp. 26672688, 2011.

[79] S. F. Mause and C. Weber, "Microparticles: protagonists of a novel communication network for intercellular information exchange," Circulation Research, vol. 107, no. 9, pp. 1047-1057, 2010.

[80] L. Deng, F. J. Blanco, H. Stevens et al., "MicroRNA-143 activation regulates smooth muscle and endothelial cell crosstalk in pulmonary arterial hypertension," Circulation Research, vol. 117, no. 10, pp. 870-883, 2015.

[81] E. Hergenreider, S. Heydt, K. Tréguer et al., "Atheroprotective communication between endothelial cells and smooth muscle cells through miRNAs," Nature Cell Biology, vol. 14, no. 3, pp. 249-256, 2012.

[82] A. D. Schecter, B. Spirn, M. Rossikhina et al., "Release of active tissue factor by human arterial smooth muscle cells," Circulation Research, vol. 87, no. 2, pp. 126-132, 2000.

[83] Y. Yuana, A. Sturk, and R. Nieuwland, "Extracellular vesicles in physiological and pathological conditions," Blood Reviews, vol. 27, no. 1, pp. 31-39, 2013.

[84] E. Campello, L. Spiezia, C. M. Radu et al., "Circulating microparticles and the risk of thrombosis in inherited deficiencies of antithrombin, protein C and protein S," Thrombosis and Haemostasis, vol. 115, no. 1, pp. 81-88, 2016.

[85] X. Tan, J. Shi, Y. Fu et al., "Role of erythrocytes and platelets in the hypercoagulable status in polycythemia vera through phosphatidylserine exposure and microparticle generation," Thrombosis and Haemostasis, vol. 109, no. 6, pp. 1025-1032, 2013. 
[86] A. Bharthuar, A. A. Khorana, A. Hutson et al., "Circulating microparticle tissue factor, thromboembolism and survival in pancreaticobiliary cancers," Thrombosis Research, vol. 132, no. 2, pp. 180-184, 2013.

[87] M. T. Sartori, A. Della Puppa, A. Ballin et al., "Circulating microparticles of glial origin and tissue factor bearing in highgrade glioma: a potential prothrombotic role," Thrombosis and Haemostasis, vol. 110, no. 2, pp. 378-385, 2013.

[88] J. A. Watts, Y.-Y. Lee, M. A. Gellar, M.-B. K. Fulkerson, S.I. Hwang, and J. A. Kline, "Proteomics of microparticles after experimental pulmonary embolism," Thrombosis Research, vol. 130, no. 1, pp. 122-128, 2012.

[89] P. Garcia Rodriguez, H. C. J. Eikenboom, M. E. T. Tesselaar et al., "Plasma levels of microparticle-associated tissue factor activity in patients with clinically suspected pulmonary embolism," Thrombosis Research, vol. 126, no. 4, pp. 345-349, 2010.

[90] J. R. Perry, "Thromboembolic disease in patients with highgrade glioma," Neuro-Oncology, vol. 14, supplement 4, pp. iv73iv80, 2012.

[91] N. Olausson, F. Mobarrez, H. Wallen, E. Westerlund, O. Hovatta, and P. Henriksson, "Microparticles reveal cell activation during IVF-a possible early marker of a prothrombotic state during the first trimester," Thrombosis and Haemostasis, vol. 116, no. 3, pp. 517-523, 2016.

[92] B. Ünlü and H. H. Versteeg, "Effects of tumor-expressed coagulation factors on cancer progression and venous thrombosis: is there a key factor?" Thrombosis Research, vol. 133, no. 2, pp. S76-S84, 2014.

[93] J. Thaler, C. Ay, N. Mackman et al., "Microparticle-associated tissue factor activity, venous thromboembolism and mortality in pancreatic, gastric, colorectal and brain cancer patients," Journal of Thrombosis and Haemostasis, vol. 10, no. 7, pp. 13631370, 2012.

[94] R. Lacroix, S. Robert, P. Poncelet, and F. Dignat-George, "Overcoming limitations of microparticle measurement by flow cytometry," Seminars in Thrombosis and Hemostasis, vol. 36, no. 8, pp. 807-818, 2010.

[95] E. Campello, L. Spiezia, C. M. Radu, and P. Simioni, "Microparticles as biomarkers of venous thromboembolic events," Biomarkers in Medicine, vol. 10, no. 7, pp. 743-755, 2016.

[96] L. Zhou, X.-L. Qi, M.-X. Xu, Y. Mao, M.-L. Liu, and H.-M. Song, "Microparticles: new light shed on the understanding of venous thromboembolism," Acta Pharmacologica Sinica, vol. 35, no. 9, pp. 1103-1110, 2014.

[97] Y. Wang, X. Lu, J. He, and W. Zhao, "Influence of erythropoietin on microvesicles derived from mesenchymal stem cells protecting renal function of chronic kidney disease," Stem Cell Research and Therapy, vol. 6, article 100, 2015.

[98] K. Tatsumi, K. Ohashi, Y. Matsubara et al., "Tissue factor triggers procoagulation in transplanted mesenchymal stem cells leading to thromboembolism," Biochemical and Biophysical Research Communications, vol. 431, no. 2, pp. 203-209, 2013.

[99] A. Steib, S.-E. Degirmenci, E. Junke et al., "Once versus twice daily injection of enoxaparin for thromboprophylaxis in bariatric surgery: effects on antifactor Xa activity and procoagulant microparticles. A randomized controlled study," Surgery for Obesity and Related Diseases, vol. 12, no. 3, pp. 613621, 2016.

[100] Z. Lysov, D. J. Dwivedi, T. J. Gould, and P. C. Liaw, "Procoagulant effects of lung cancer chemotherapy: impact on microparticles and cell-free DNA," Blood Coagulation and Fibrinolysis, vol. 28, no. 1, pp. 72-82, 2017.
[101] R. E. Tilley, T. Holscher, R. Belani, J. Nieva, and N. Mackman, "Tissue factor activity is increased in a combined platelet and microparticle sample from cancer patients," Thrombosis Research, vol. 122, no. 5, pp. 604-609, 2008.

[102] J. P. Wrobel, B. R. Thompson, and T. J. Williams, "Mechanisms of pulmonary hypertension in chronic obstructive pulmonary disease: a pathophysiologic review," Journal of Heart and Lung Transplantation, vol. 31, no. 6, pp. 557-564, 2012.

[103] M. A. Albayati, S. P. Grover, P. Saha, B. A. Lwaleed, B. Modarai, and A. Smith, "Postsurgical inflammation as a causative mechanism of venous thromboembolism," Seminars in Thrombosis and Hemostasis, vol. 41, no. 6, pp. 615-620, 2015.

[104] C. Venetz, J. Labarère, D. Jiménez, and D. Aujesky, "White blood cell count and mortality in patients with acute pulmonary embolism," American Journal of Hematology, vol. 88, no. 8, pp. 677-681, 2013.

[105] E. G. Bovill, D. E. Bild, G. Heiss et al., "White blood cell counts in persons aged 65 years or more from the cardiovascular health study: correlations with baseline clinical and demographic characteristics," American Journal of Epidemiology, vol. 143, no. 11, pp. 1107-1115, 1996.

[106] J. Y. Jo, M. Y. Lee, J. W. Lee, B. H. Rho, and W.-I. Choi, "Leukocytes and systemic inflammatory response syndrome as prognostic factors in pulmonary embolism patients," $B M C$ Pulmonary Medicine, vol. 13, no. 1, article 74, 8 pages, 2013.

[107] H. Kundi, A. Balun, H. Cicekcioglu et al., "The relation between platelet-to-lymphocyte ratio and Pulmonary Embolism Severity Index in acute pulmonary embolism," Heart and Lung, vol. 44, no. 4, pp. 340-343, 2015.

[108] K. Soylu, Ö. Gedikli, A. Ekşi et al., "Neutrophil-to-lymphocyte ratio for the assessment of hospital mortality in patients with acute pulmonary embolism," Archives of Medical Science, vol.12, no. 1, pp. 95-100, 2016.

[109] J. A. Watts, M. A. Gellar, L. K. Stuart, M. Obraztsova, and J. A. Kline, "Proinflammatory events in right ventricular damage during pulmonary embolism: effects of treatment with ketorolac in rats," Journal of Cardiovascular Pharmacology, vol. 54, no. 3, pp. 246-252, 2009.

[110] J. Zagorski, J. Debelak, M. Gellar, J. A. Watts, and J. A. Kline, "Chemokines accumulate in the lungs of rats with severe pulmonary embolism induced by polystyrene microspheres," The Journal of Immunology, vol. 171, no. 10, pp. 5529-5536, 2003.

[111] J. A. Watts, M. R. Marchick, and J. A. Kline, "Right ventricular heart failure from pulmonary embolism: key distinctions from chronic pulmonary hypertension," Journal of Cardiac Failure, vol. 16, no. 3, pp. 250-259, 2010.

[112] M. M. Orde, R. Puranik, P. L. Morrow, and J. Duflou, "Myocardial pathology in pulmonary thromboembolism," Heart, vol. 97, no. 20, pp. 1695-1699, 2011.

[113] M. Cazzola, L. Calzetta, G. Bettoncelli, L. Novelli, C. Cricelli, and P. Rogliani, "Asthma and comorbid medical illness," European Respiratory Journal, vol. 38, no. 1, pp. 42-49, 2011.

[114] C. J. Majoor, P. W. Kamphuisen, A. H. Zwinderman et al., "Risk of deep vein thrombosis and pulmonary embolism in asthma," European Respiratory Journal, vol. 42, no. 3, pp. 655-661, 2013.

[115] W.-S. Chung, C.-L. Lin, F.-M. Ho et al., "Asthma increases pulmonary thromboembolism risk: a nationwide population cohort study," European Respiratory Journal, vol. 43, no. 3, pp. 801-807, 2014.

[116] M. M. Tomasiak-Lozowska, T. Rusak, T. Misztal, A. BodzentaLukaszyk, and M. Tomasiak, "Reduced clot retraction rate and 
altered platelet energy production in patients with asthma," Journal of Asthma, vol. 53, no. 6, pp. 589-598, 2016.

[117] L. Bertoletti, S. Quenet, P. Mismetti et al., "Clinical presentation and outcome of venous thromboembolism in COPD," European Respiratory Journal, vol. 39, no. 4, pp. 862-868, 2012.

[118] W.-J. Chen, C.-C. Lin, C.-Y. Lin et al., "Pulmonary embolism in chronic obstructive pulmonary disease: a population-based cohort study," Journal of Chronic Obstructive Pulmonary Disease, vol. 11, no. 4, pp. 438-443, 2014.

[119] E. E. Akpinar, D. Hoşgün, S. Akpinar, G. K. Ataç, B. Doğanay, and M. Gülhan, "Incidence of pulmonary embolism during COPD exacerbation," Jornal Brasileiro de Pneumologia, vol. 40, no. 1, pp. 38-45, 2014.

[120] M. Bahloul, A. Chaari, A. Tounsi et al., "Incidence and impact outcome of pulmonary embolism in critically ill patients with severe exacerbation of chronic obstructive pulmonary diseases," Clinical Respiratory Journal, vol. 9, no. 3, pp. 270-277, 2015.

[121] C. Garlanda, C. A. Dinarello, and A. Mantovani, "The interleukin-1 family: back to the future," Immunity, vol. 39, no. 6, pp. 1003-1018, 2013.

[122] S. Sanjabi, L. A. Zenewicz, M. Kamanaka, and R. A. Flavell, "Anti-inflammatory and pro-inflammatory roles of TGF- $\beta$, IL10, and IL-22 in immunity and autoimmunity," Current Opinion in Pharmacology, vol. 9, no. 4, pp. 447-453, 2009.

[123] F. O. Martinez and S. Gordon, "The M1 and M2 paradigm of macrophage activation: time for reassessment," F1000Prime Reports, vol. 6, article 13, 2014.

[124] T. A. Fuchs, A. Brill, and D. D. Wagner, "Neutrophil extracellular trap (NET) impact on deep vein thrombosis," Arteriosclerosis, Thrombosis, and Vascular Biology, vol. 32, no. 8, pp. 17771783, 2012.

[125] K. Martinod and D. D. Wagner, "Thrombosis: tangled up in NETs," Blood, vol. 123, no. 18, pp. 2768-2776, 2014.

[126] L. Ma and A. Dorling, "The roles of thrombin and proteaseactivated receptors in inflammation," Seminars in Immunopathology, vol. 34, no. 1, pp. 63-72, 2012.

[127] E. Niccolai, G. Emmi, D. Squatrito et al., "Microparticles: bridging the gap between autoimmunity and thrombosis," Seminars in Thrombosis and Hemostasis, vol. 41, no. 4, pp. 413-422, 2015.

[128] H. Shen, D. Kreisel, and D. R. Goldstein, "Processes of sterile inflammation," Journal of Immunology, vol. 191, no. 6, pp. 28572863, 2013.

[129] Y. Xiang, C. Yan, X. Guo et al., "Host-derived, pore-forming toxin-like protein and trefoil factor complex protects the host against microbial infection," Proceedings of the National Academy of Sciences of the United States of America, vol. 111, no. 18, pp. 6702-6707, 2014

[130] Y. Xiang, X. Wang, C. Yan et al., "Adenosine- $5^{\prime}$-triphosphate (ATP) protects mice against bacterial infection by activation of the NLRP3 inflammasome," PLoS ONE, vol. 8, no. 5, Article ID e63759, 2013.

[131] L. R. Villegas, D. Kluck, C. Field et al., "Superoxide dismutase mimetic, MnTE-2-PyP, attenuates chronic hypoxia-induced pulmonary hypertension, pulmonary vascular remodeling, and activation of the NALP3 inflammasome," Antioxidants and Redox Signaling, vol. 18, no. 14, pp. 1753-1764, 2013.

[132] J. Bělohlávek, V. Dytrych, and A. Linhart, "Pulmonary embolism, part I: epidemiology, risk factors and risk stratification, pathophysiology, clinical presentation, diagnosis and nonthrombotic pulmonary embolism," Experimental and Clinical Cardiology, vol. 18, no. 2, pp. 129-138, 2013.
[133] J. Ryan, K. Bloch, and S. L. Archer, "Rodent models of pulmonary hypertension: harmonisation with the world health organisation's categorisation of human $\mathrm{PH}$, , International Journal of Clinical Practice. Supplement, no. 172, pp. 15-34, 2011. 


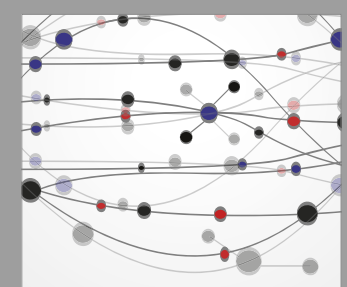

The Scientific World Journal
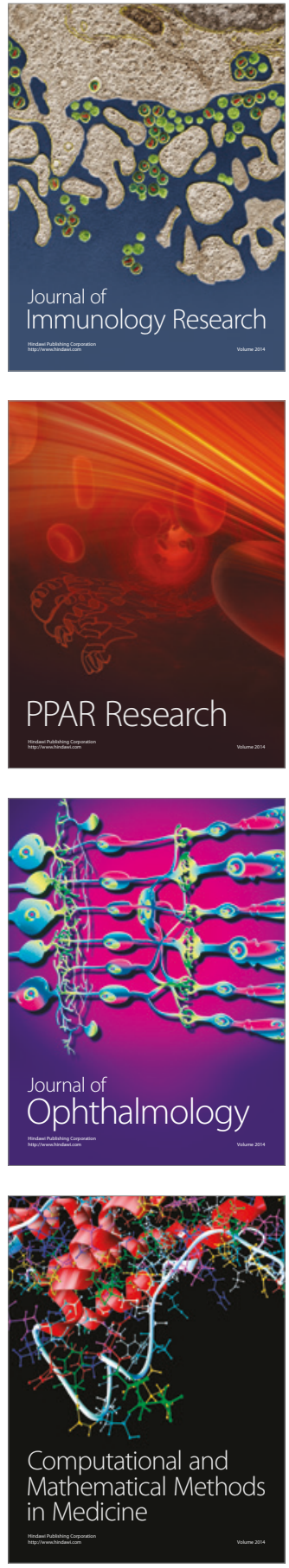

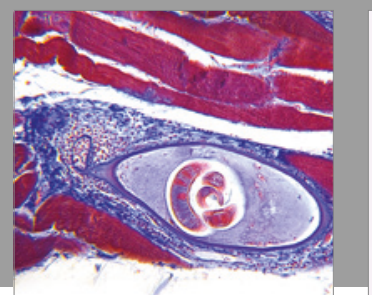

Gastroenterology Research and Practice
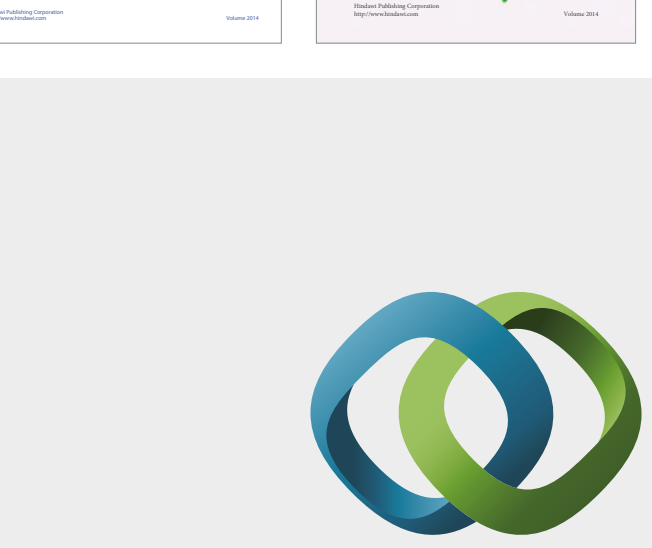

\section{Hindawi}

Submit your manuscripts at

https://www.hindawi.com
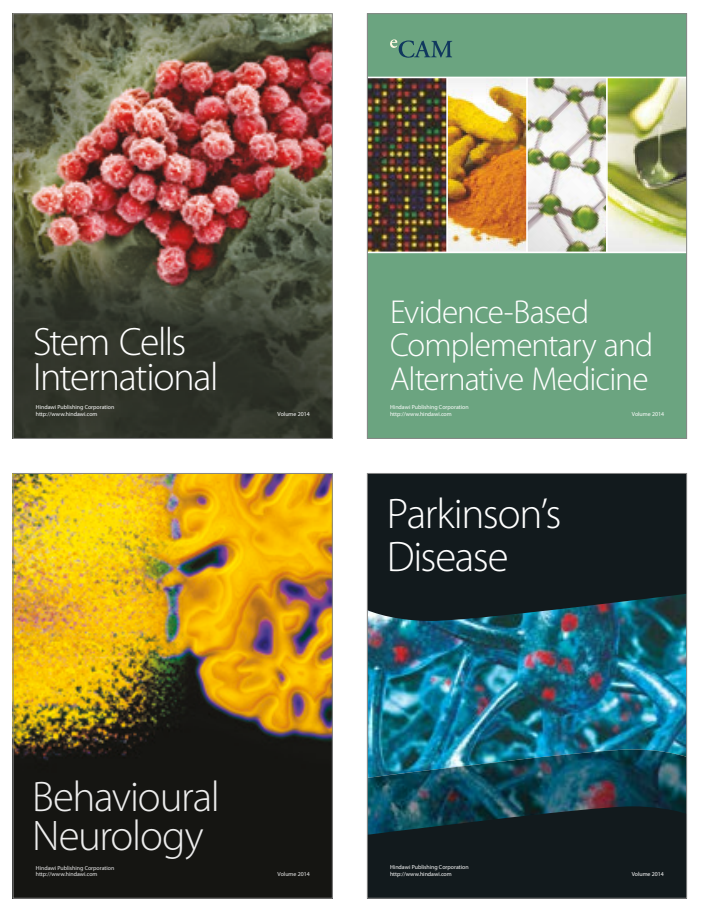
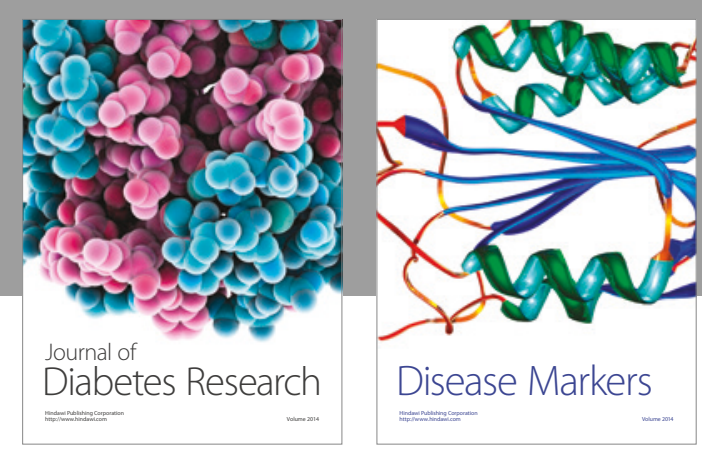

Disease Markers
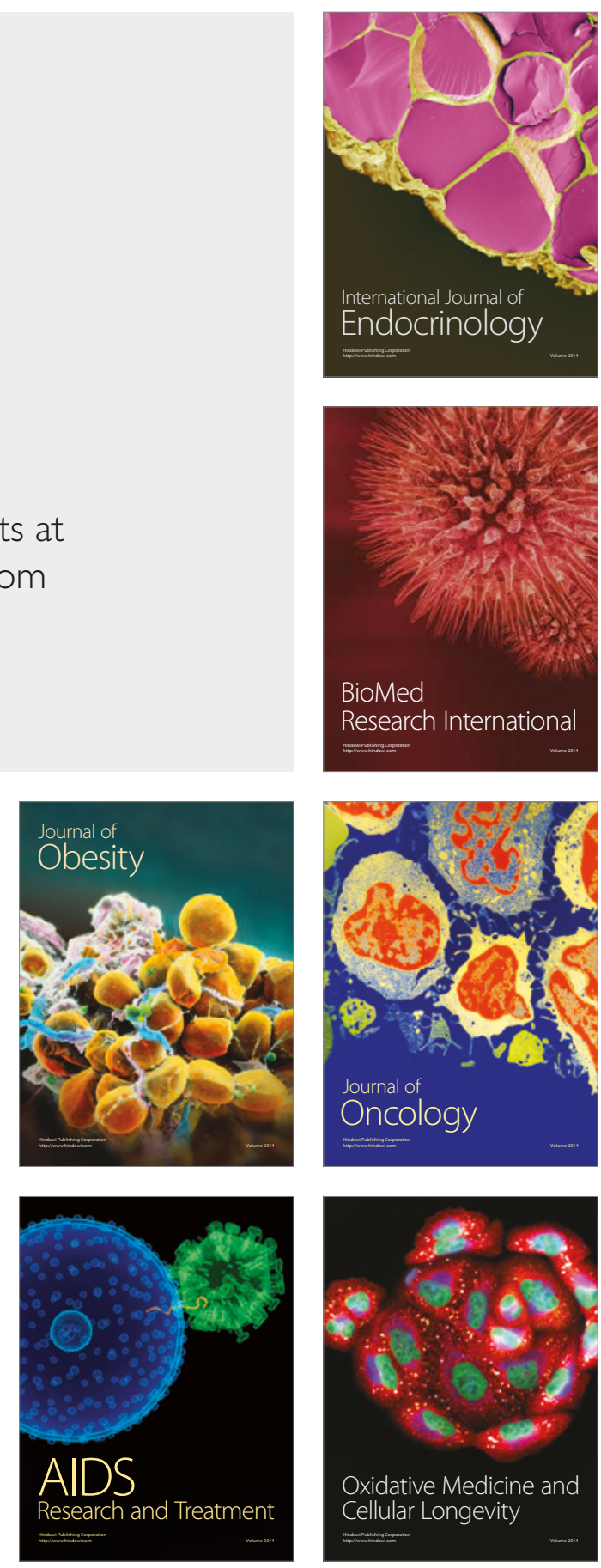